\title{
Accuracy of the urine UCA1 for diagnosis of bladder cancer: a meta-analysis
}

\author{
Xiangrong Cui ${ }^{1,2,4, *}$, Xuan Jing ${ }^{3, *}$, Chunlan Long ${ }^{1,4,5}$, Qin $\mathbf{Y i}^{1,4,5}$, Jie Tian ${ }^{6}$ and Jing \\ Zhu $^{1,4,5}$ \\ ${ }^{1}$ Pediatric Research Institute, Children's Hospital of Chongqing Medical University, Ministry of Education Key Laboratory of \\ Child Development and Disorders, Chongqing, China \\ 2 Reproductive Medicine Center, Children's Hospital of Shanxi and Women's Health Center of Shanxi, Affiliate of Shanxi \\ Medical University, Taiyuan, China \\ ${ }^{3}$ Clinical Laboratory, Shanxi Province People's Hospital, Affiliate of Shanxi Medical University, Taiyuan, China \\ ${ }^{4}$ China International Science and Technology Cooperation Base of Child Development and Critical Disorders, Chongqing, \\ China \\ ${ }^{5}$ Chongqing Key Laboratory of Pediatrics, Chongqing, China \\ ${ }^{6}$ Cardiovascular Department (Internal Medicine), Children's Hospital of Chongqing Medical University, Chongqing, China \\ * These authors have contributed equally to this work \\ Correspondence to: Jing Zhu, email:412232858@qq.com
}

Keywords: UCA1, biomarker, bladder cancer, noninvasive diagnosis, urinary marker

Received: January 30, $2017 \quad$ Accepted: March 11, $2017 \quad$ Published: March 22, 2017

Copyright: Cui et al. This is an open-access article distributed under the terms of the Creative Commons Attribution License (CC-BY), which permits unrestricted use, distribution, and reproduction in any medium, provided the original author and source are credited.

\section{ABSTRACT}

Urine UCA1 has been reported as a potential novel diagnostic biomarker for bladder cancer in several studies, but their results are inconsistent. As a result of this, a diagnostic meta-analysis to assess the diagnostic performance of urine UCA1 in detecting bladder cancer was conducted. A systematic electronic and manual search was performed for relevant literatures through PubMed, Cochrane library, Chinese Wan Fang and the China National Knowledge Infrastructure (CNKI) databases up to December 30, 2016. The quality of the studies included in this meta-analysis was assessed using the Quality Assessment of Diagnostic Accuracy Studies (QUADAS-2) tool. All analyses were conducted using stata12.0 software. Six studies collectively included 578 bladder cancer patients and 562 controls met the eligible criteria. The overall diagnostic accuracy was measured by the following: sensitivity 0.81 (95\% $\mathrm{CI}=0.75-0.86)$, specificity $0.86(95 \% \mathrm{CI}=0.73-0.93)$, positive likelihood ratio 5.85 (95\% CI $=2.72-12.57)$, negative likelihood $0.22(95 \% \mathrm{CI}=0.15-0.32)$, diagnostic odds ratio $27.01(95 \% \mathrm{CI}=\mathbf{8 . 6 9 - 8 3 . 9 7 )}$, and area under the curve $0.88(95 \% \mathrm{CI}=$ 0.85-0.91). Meta-regression analysis suggested that ethnicity significantly accounted for the heterogeneity of sensitivity. Deeks' funnel plot asymmetry test $(P=0.33)$ suggested no potential publication bias. According to our results, urine UCA1 has greater diagnostic value in diagnosing bladder cancer, however further research studies with more well-designed and large sample sizes are required to confirm our findings.

\section{INTRODUCTION}

Bladder cancer $(\mathrm{BC})$ is the second most common urogenital malignancy, with $50 \%$ recurrence rate and $15-40 \%$ growing into muscle invasive disease $[1,2]$. Early diagnosis and reliable follow-up for recurrences after conservative treatment are extremely important for improving treatment of $\mathrm{BC}$ [3-5]. Cystoscopy is currently considered the golden standard for diagnosing bladder cancer, often combined with urinary cytology [6]. However, the invasive nature of cystoscopy and low sensitivity of cytology restrict their application in the early 
diagnosis of $\mathrm{BC}[7,8]$. Therefore, exploring more reliable non-invasive detection of new or recurrent bladder cancer is the need of the hour.

Long non-coding RNAs (lncRNAs) are a class of transcribed RNA molecules with more than 200 nucleotides in length and lack protein coding function [9-11]. Accumulating studies have shown that the dysregulation of lncRNAs was closely related to oncogenesis, metastasis, and prognosis in cancers [1214]. Their expression patterns in various cancer types have been extensively identified, and many of these lncRNAs might be used as independent biomarkers for tumor diagnosis and treatment [15-17]. Recently, the lncRNAs, urothelial carcinoma associated 1 (UCA1), was firstly identified in the tissue and urine in 2006 and was found that it might play a crucial role in BC progression and embryogenesis [18]. In addition, UCA1 was also detected as a very sensitive and specific urine marker in $\mathrm{BC}$ diagnosis [19, 20].

Despite many studies have demonstrated the potential of urine UCA1 as a novel diagnostic marker for $\mathrm{BC}$, the previous studies have been limited by relatively small sample size recruited in the individual studies, and no previous published meta-analysis have addressed this research question. Thus, we carried out this meta-analysis to review and assess the overall diagnostic test accuracy of UCA1 for BC diagnosis.

\section{RESULTS}

\section{Study characteristics}

As shown in the flow diagram (Figure 1), 351 potentially relevant articles were searched in the databases. After a detailed evaluation, 6 studies [18, 19, 21-24] were included in the current meta-analysis. The main characteristics of included studies were summarized in Table 1 ranging from 2003 to 2015 . The total number of patients and controls were 578 and 562, respectively. Among the 6 studies, 3 studies were conducted in China [18, 23, 24], 1 in India [21], 1in Belgium [19], 1 in Egypt [22], which means three studies were conducted in Chinese populations, three studies were conducted in non-Chinese population. All the included studies used urine sediment as specimens and used reverse transcription polymerase chain reaction (RT-PCR) or quantitative RT-PCR method to determine the expression of UCA1 in urine sediment.

\section{Quality assessment of studies}

The results of the QUADAS-2 study quality assessment were shown in Figure 2. The majority of all included articles in current meta-analysis met most items in QUADAS-2, suggesting that the overall quality of included studies were of moderate-high.

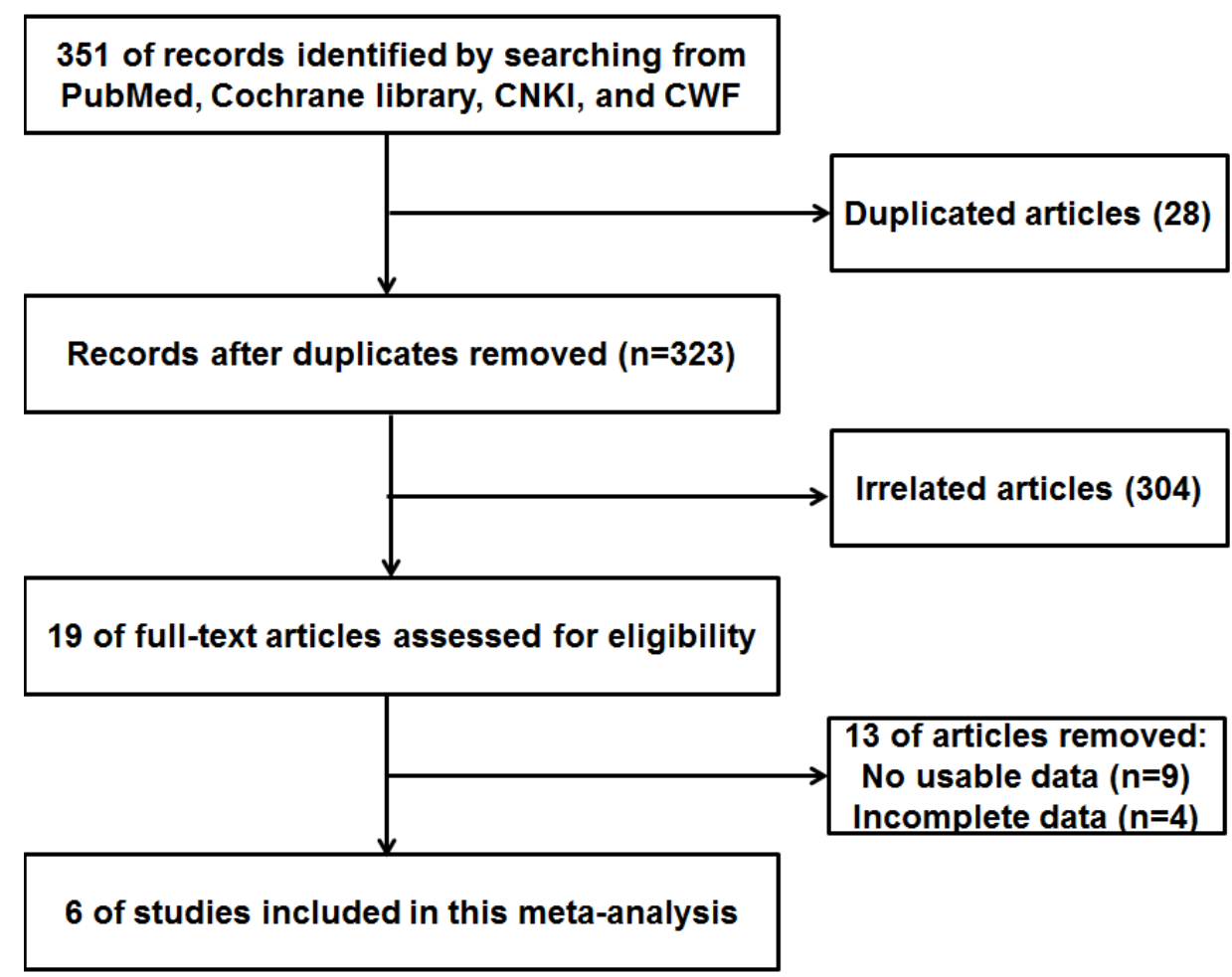

Figure 1: The flow diagram of this meta-analysis. 
Table 1: Characteristics of the included studies

\begin{tabular}{|c|c|c|c|c|c|c|c|c|c|c|c|}
\hline \multirow{2}{*}{ Study ID } & \multirow{2}{*}{ Country } & \multirow{2}{*}{ Ethnicity } & \multicolumn{2}{|c|}{ Sample size } & \multirow{2}{*}{ Cancer } & \multirow{2}{*}{ Specimen } & \multirow{2}{*}{ Method } & \multicolumn{4}{|c|}{ Diagnostic power } \\
\hline & & & Case & Control & & & & $\overline{T P}$ & FP & FN & TN \\
\hline Wang XS, 2006 & China & Chinese & 94 & 85 & $\mathrm{BC}$ & urine & RT-PCR & 76 & 7 & 18 & 78 \\
\hline Zhang Z, 2012 & China & Chinese & 180 & 144 & $\mathrm{BC}$ & urine & RT-PCR & 152 & 11 & 28 & 133 \\
\hline Li F, 2012 & China & Chinese & 24 & 50 & $\mathrm{BC}$ & urine & qRT-PCR & 21 & 20 & 3 & 30 \\
\hline Srivastava AK, 2014 & India & non-Chinese & 117 & 74 & $\mathrm{BC}$ & urine & qRT-PCR & 93 & 15 & 24 & 59 \\
\hline Milowich D, 2015 & Belgium & non-Chinese & 69 & 93 & $\mathrm{BC}$ & urine & RT-PCR & 48 & 27 & 21 & 66 \\
\hline Eissa S, 2015 & Egypt & non-Chinese & 94 & 116 & $\mathrm{BC}$ & urine & qRT-PCR & 86 & 4 & 8 & 112 \\
\hline
\end{tabular}

BC: bladder carcinoma; TP: true positive; FP: false positive; TN: true negative; FN: false negative

\section{Diagnostic accuracy}

The forest plot of data from included articles on sensitivity and specificity for UCA1 assay in diagnosing bladder cancer is shown in Figure 3. Significant heterogeneity was found for both sensitivity $\left(I^{2}=66 \%\right.$,

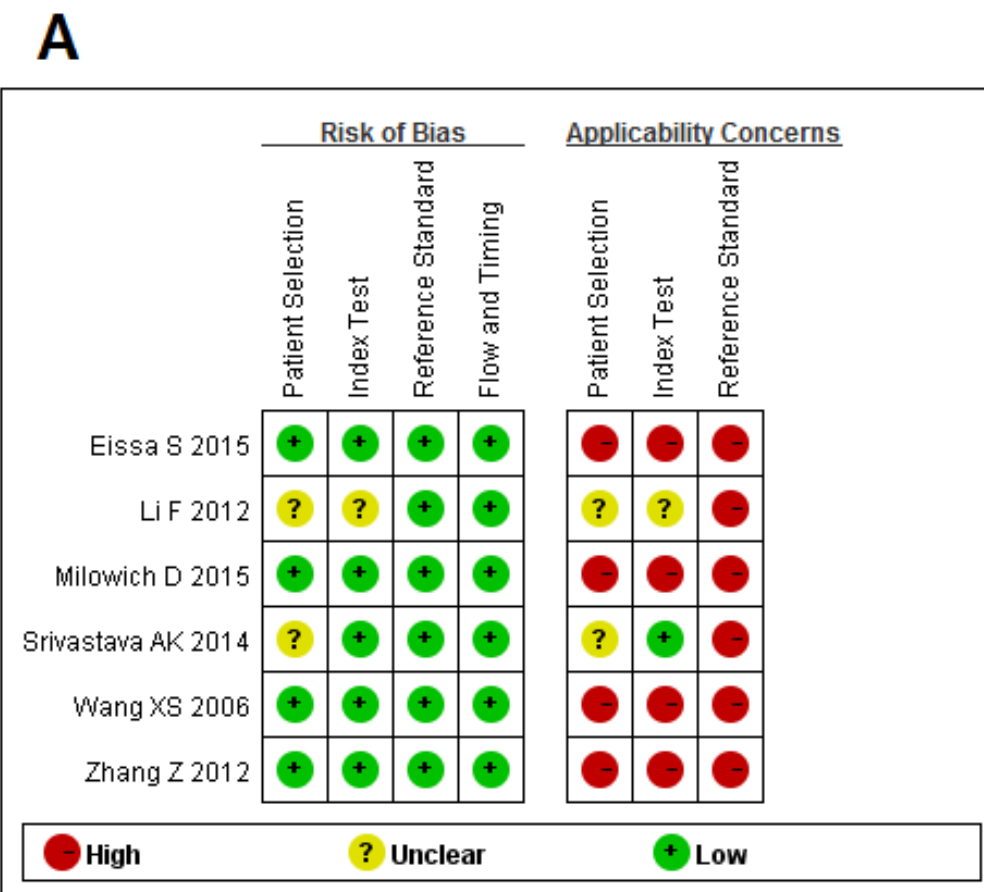

B

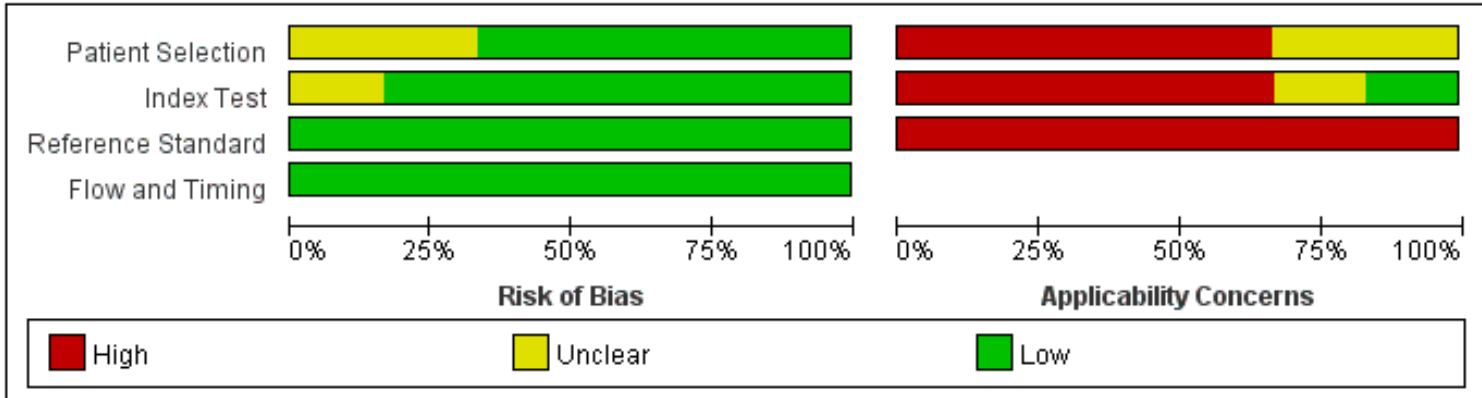

Figure 2: Quality assessments of included studies by using the QUADAS-2 tool. A. risk of bias summary: review authors' judgments about each risk of bias item for each included study; B. risk of bias graph: review authors' judgments about each item presented as percentages across all included studies. 


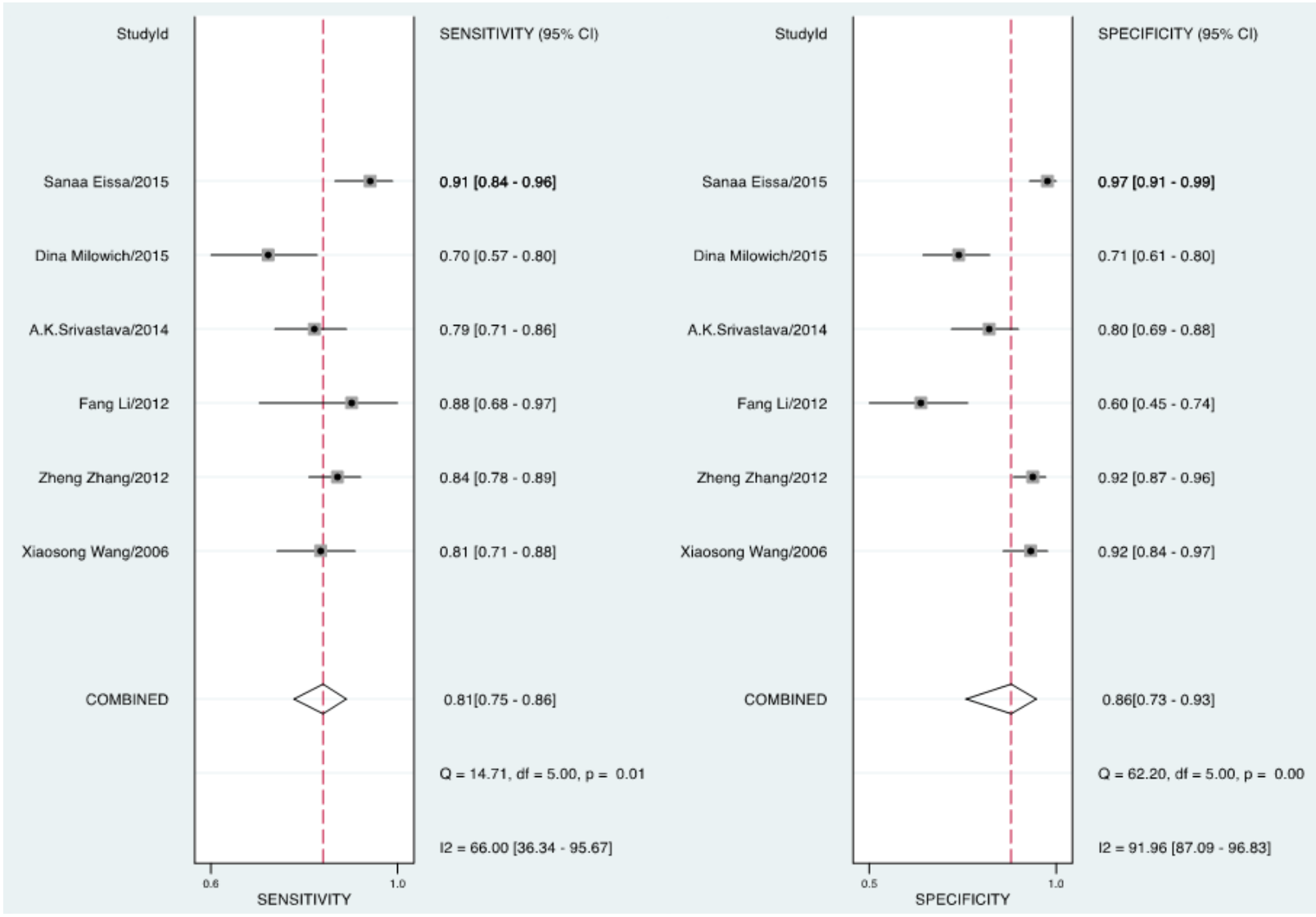

Figure 3: Forest plots of the sensitivity and specificity for UCA1 in the diagnosis of bladder cancer.

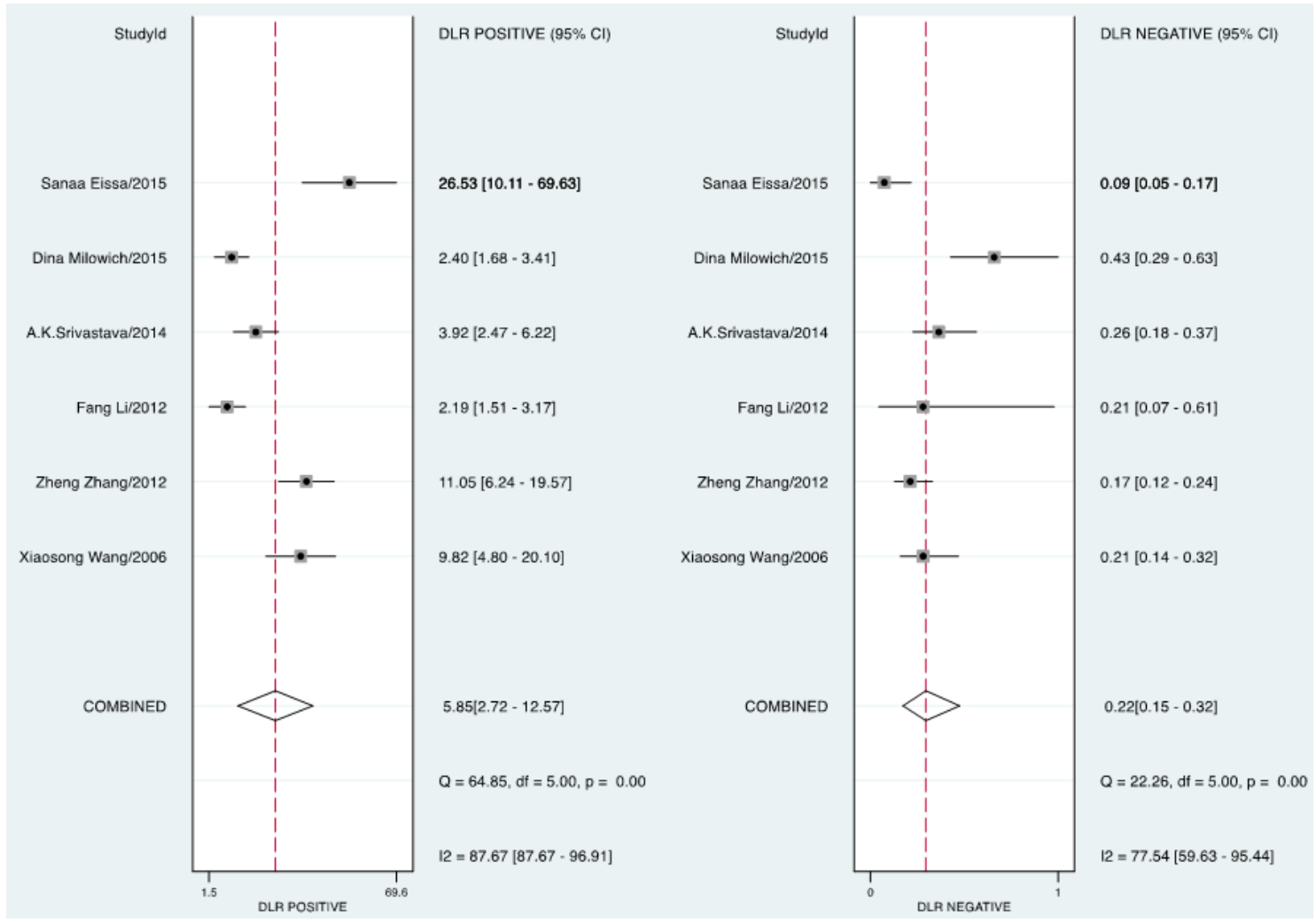

Figure 4: Forest plots of estimated positive likelihood ratio (PLR) and negative likelihood ratio (NLR) for urine UCA1 in the diagnosis of bladder cancer. 
$95 \% \mathrm{CI}=36.34 \%-95.67 \%)$ and specificity $\left(I^{2}=91.96 \%\right.$, $95 \% \mathrm{CI}=87.09 \%-96.83 \%$ ). Therefore, the random effects model was applied for the further analysis. Overall, the sensitivity and specificity for the pooled data were 0.81 $(95 \% \mathrm{CI}=0.75-0.86)$ and $0.86(95 \% \mathrm{CI}=0.73-0.93)$, respectively. In addition, the pooled PLR was $5.85(95 \%$ $\mathrm{CI}=2.72-12.57)$, the NLR was $0.22(95 \% \mathrm{CI}=0.15-0.32)$, and the DOR was $27.01(95 \% \mathrm{CI}=8.69-83.97)$ (Figure 4 and 5). The SROC curve for the 6 included studies in shown in Figure 6. The AUC of urine UCA1 tests was $0.88(95 \% \mathrm{CI}=0.85-0.91)$, thereby implying a relatively high diagnostic value. To evaluate the clinical utility of the index test, a Fagan's Nomogram was performed to predict the increasing inerrability about a positive diagnosis by using the value of the test and it is used for estimating post-test probabilities (Figure 7).

\section{Meta-regression and subgroup analysis}

The sources of potential heterogeneity in sensitivity and specificity were explored by univariate metaregression analysis and subgroup analysis. As shown in Figure 8 , in the 3 Chinese studies, the pooled sensitivity and specificity was $0.81 \%(95 \% \mathrm{CI}=0.73-0.89)$ and $0.85 \%$ (95\% CI $=0.71-1.00)$ respectively, and in 3 nonChinese studies, the pooled sensitivity and specificity was
$0.81(95 \% \mathrm{CI}=0.74-0.89)$ and $0.87(95 \% \mathrm{CI}=0.73-1.00)$ respectively. The ethnicity significantly accounted for the heterogeneity of sensitivity.

\section{Threshold effect}

Differences in cut-off values lead to the threshold effect. When there is a threshold effect, an inverse correlation is demonstrated among the sensitivity and specificity, leading to a typical 'shoulder arm' of the ROC plane distribution. Spearman correlation analysis is also suggests a strong positive correlation. In the current study, the representation of the sensitivity against the specificity of each study shown in an ROC plane (Figure 9), displayed a non-typical shoulder arm appearance, indicating the absence of the threshold effect. In addition, the calculated Spearman correlation coefficient value was -0.429 ( $P=$ 0.397 ), also suggesting that there was no threshold effect.

\section{Publication bias}

In our meta-analysis, Deeks' funnel plot asymmetry test was conducted to evaluate potential publication bias (Figure 10). No significant publication bias existed among the studies $(P=0.33)$.

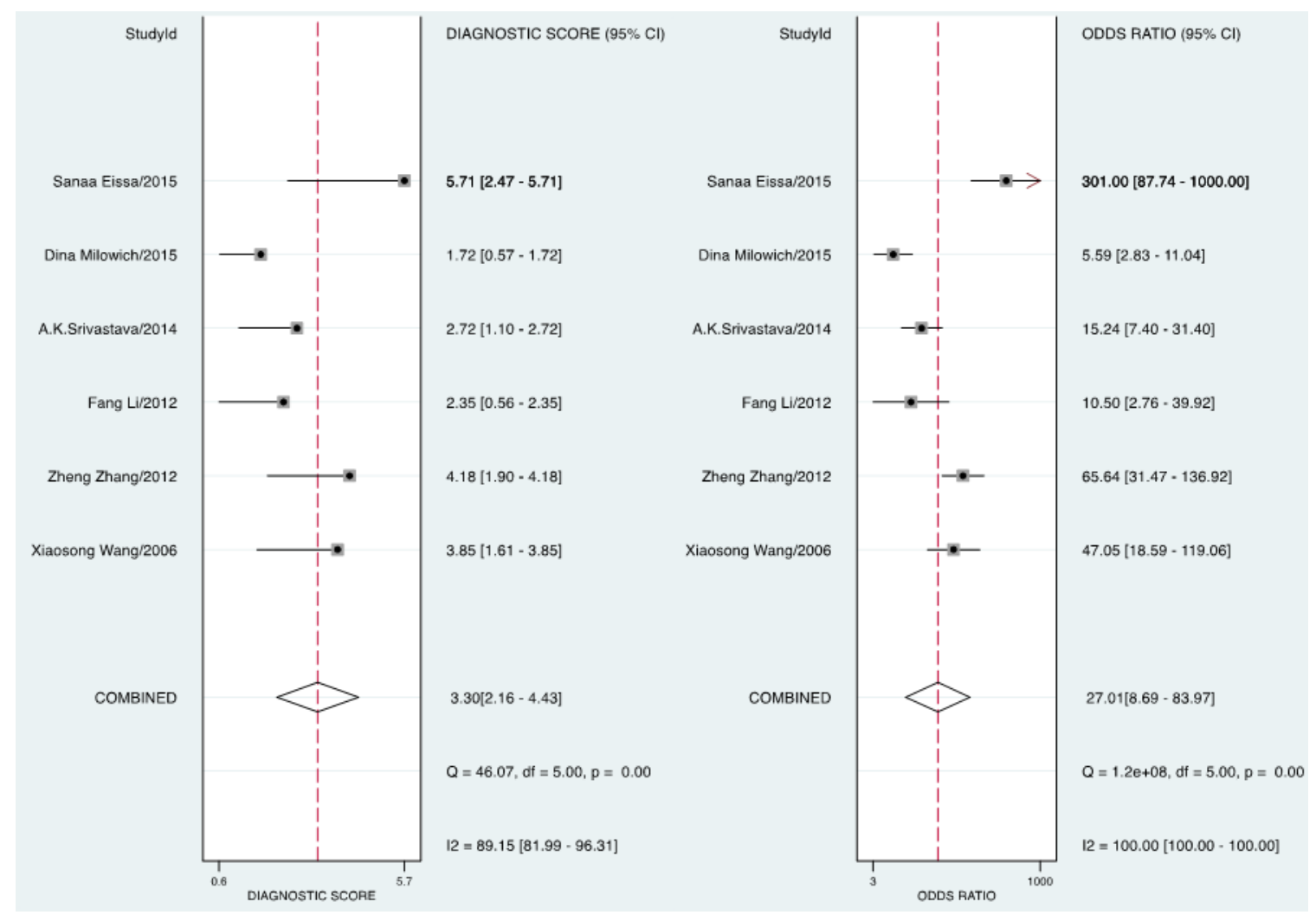

Figure 5: Forest plots of estimated pooled diagnostic odds ratio (DOR) for urine UCA1 in the diagnosis of bladder cancer. 


\section{DISCUSSION}

Bladder cancer is one of the most common male genitourinary tumors [25-27]. To date, although bladder cancer can be initial diagnosed by screening cystoscopy, random bladder biopsies, and voided urinary cytology, the first two methods are invasive and uncomfortable, and the low sensitivity and high variability of cytology creates a challenge that limits its application in the early diagnosis of bladder cancer due to inter-observer reproducibility [2830]. Thus, searching a feasible, reliable, and minimally noninvasive method to detect new or recurrent bladder cancer is the need of the hour.

In current meta-analysis, we conducted the first diagnostic meta-analysis to assess the accuracy of urine UCA1 as a biomarker for bladder cancer. An AUC of 0.88 (95\% CI, 0.85-0.91), with pooled SEN of 0.81 (95\% CI, $0.75-0.86)$ and SPE of 0.86 (95\% CI, 0.73-0.93) showed that UCA1 in voided urine sediment may be a promising biomarker to discriminate bladder cancer patients from normal bladder. As a prevalence-independent indicator, DOR value could indicate the degree of the association between diagnostic results and disease. The pooled DOR of 27.01 (95\% CI, 8.69-83.97) suggested that the overall accuracy of UCA1 for the diagnosis of bladder cancer is credible.

The likelihood ratio (LR), including PLR and NLR, could also reflect the diagnostic accuracy. When positive likelihood ratio $>10$ or negative likelihood ratio $<0.1$, the likelihood of diagnosis or exclusion of a disease increased significantly. Nevertheless, in our meta-analysis, a pooled PLR of 5.85 (95\% CI, 2.72-12.57) and NLR 0.22 (95\% C, 0.15-0.32) indicated that patients with bladder cancer have an $~ 5.86$-fold higher chance of testing positive using UCA1 compared with controls and 33\% individuals with bladder cancer have an negative result.

LR and post-test probabilities are correlation with clinicians, due to they provide information about the likelihood of a patient with a positive or negative test actually exhibiting bladder cancer. From the Fagan's Nomogram, we found that when a pre-test probability of $20 \%$ was specified, the post-test probability positivity would raise to $59 \%$ with a positive likelihood ratio of 6 , and the post-test probability negativity would decreased

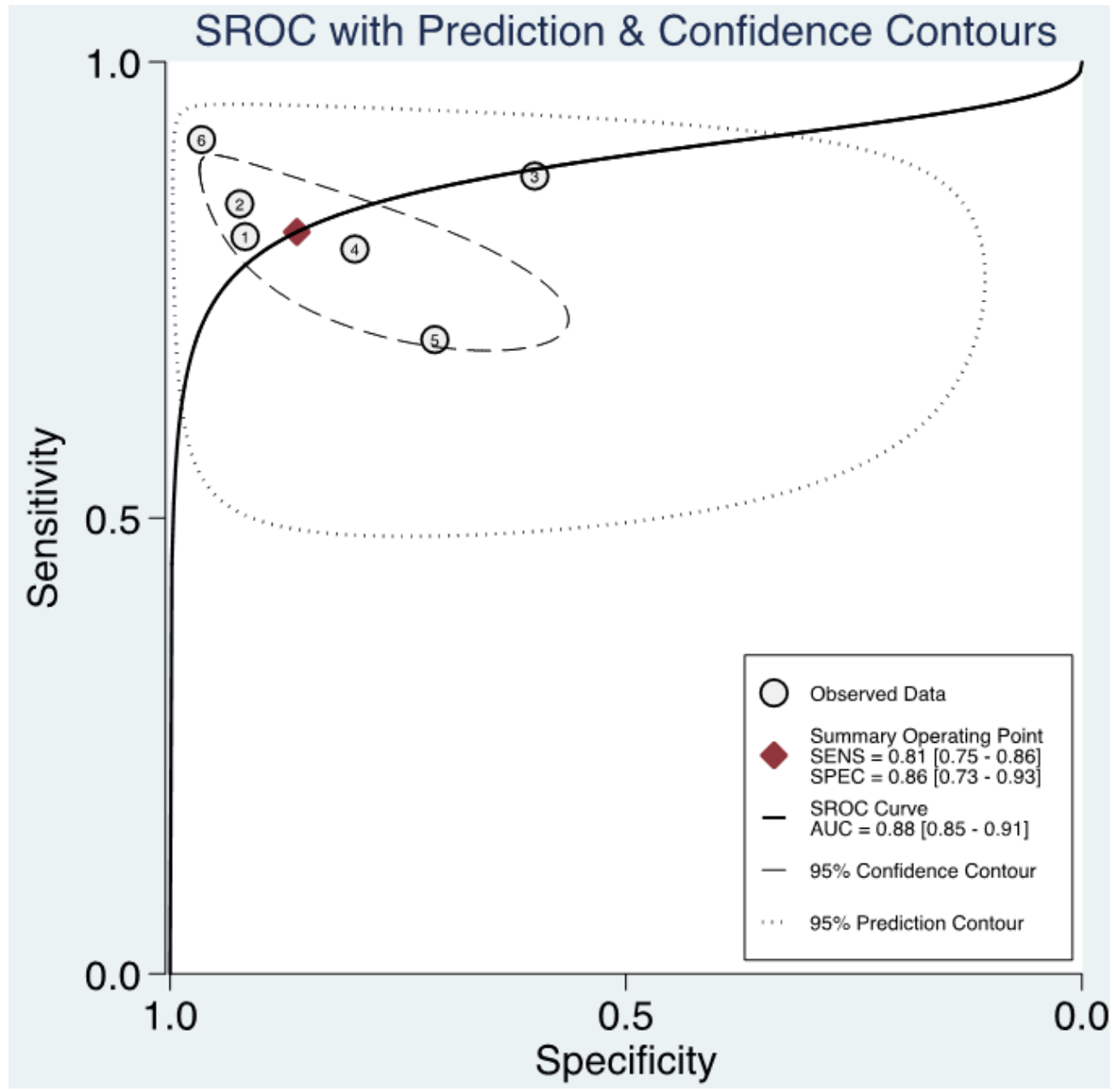

Figure 6: Summary receiver operating characteristic graph of included studies. 


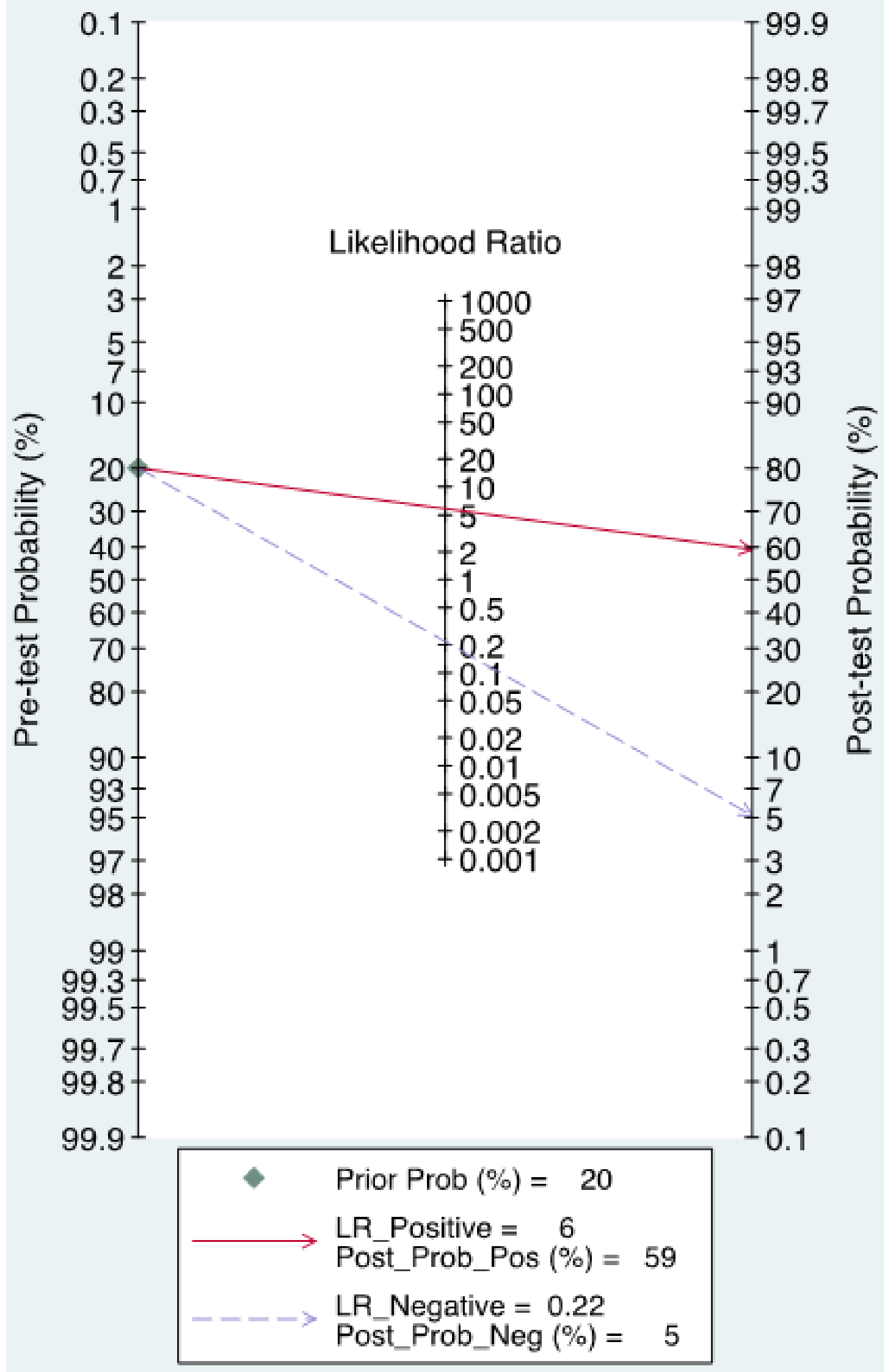

Figure 7: Fagan's nomogram for calculation of post-test probabilities. 
to $5 \%$ with a negative likelihood ratio was 0.22 . These outcomes suggest a stable value for UCA1 in the diagnosis of bladder cancer.
Heterogeneity is a potential obstacle when interpreting the results for meta-analysis, which should be seriously considered [31]. Although rigorous approach

\section{Univariable Meta-regression \& Subgroup Analyses}
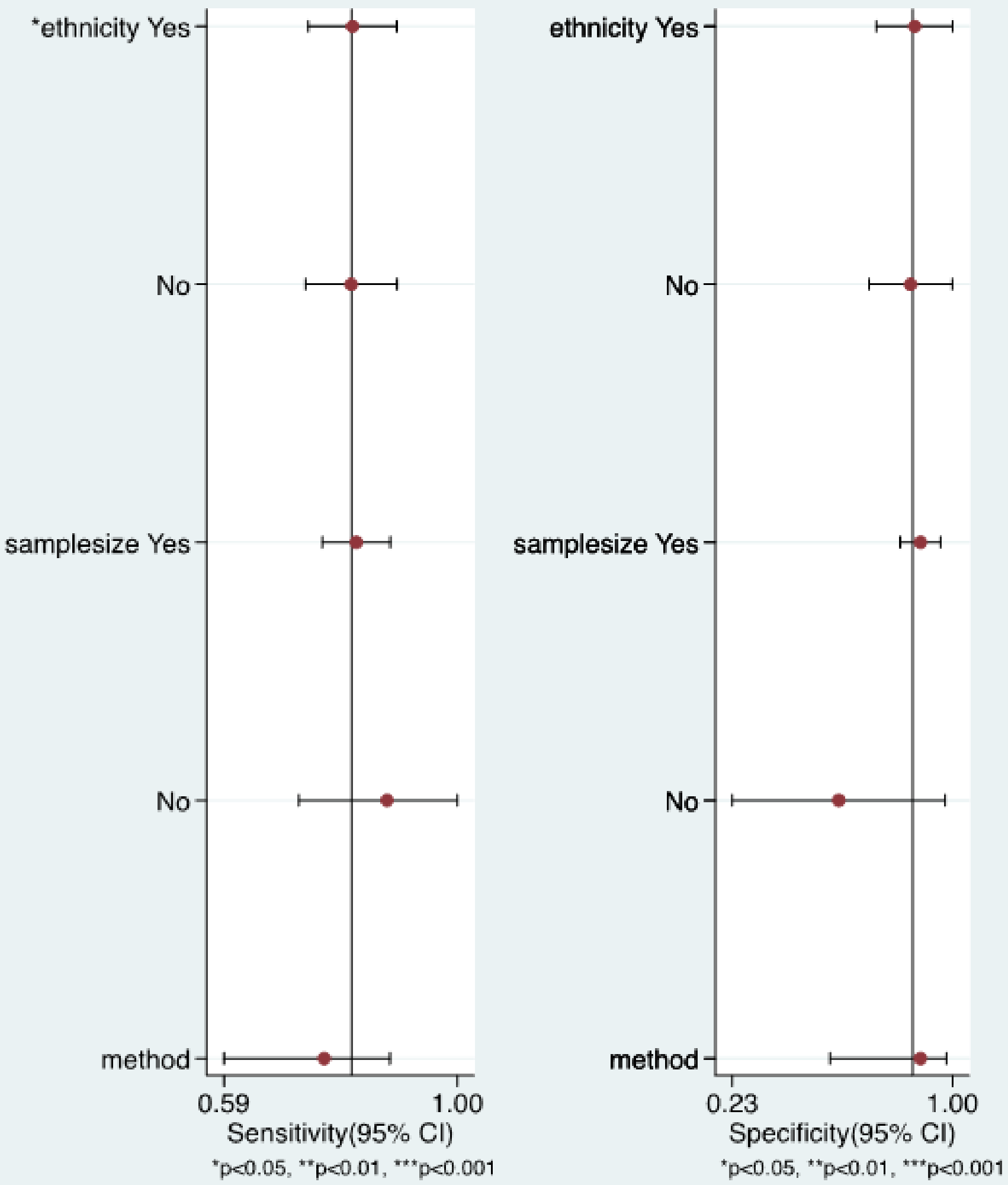

Figure 8: Univariable meta-regression and subgroup analysis. 
has been adopted to retrieve documents, there was still potential heterogeneity in our current study. One source of heterogeneity is the threshold effect, which arises due to variable cut-off values adopted in different studies to determine whether the results were negative or positive. The ROC plane displayed a non-typical shoulder arm appearance, indicating the absence of the threshold effect.
In addition, the calculated Spearman correlation coefficient value was $-0.429(P=0.397)$, also suggesting that threshold effect is not the cause of heterogeneity. Then, we found that sample size and ethnicity may have partially led to such heterogeneity. Therefore, subgroup analyses were performed to evaluate the contribution of the factors above sources of potential heterogeneity in sensitivity and

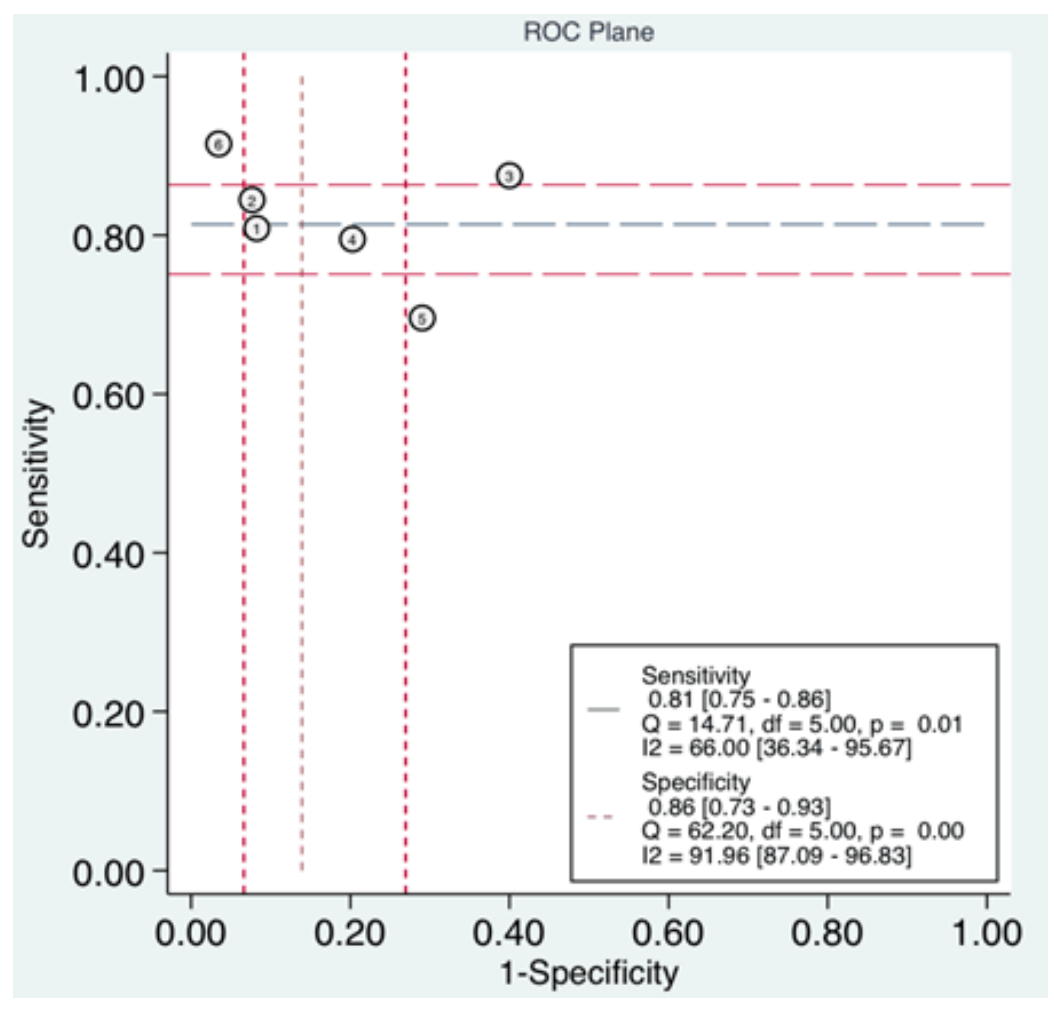

Figure 9: Receiver operating characteristics (ROC) space for the assessment of the threshold effect in UCA1 assays.

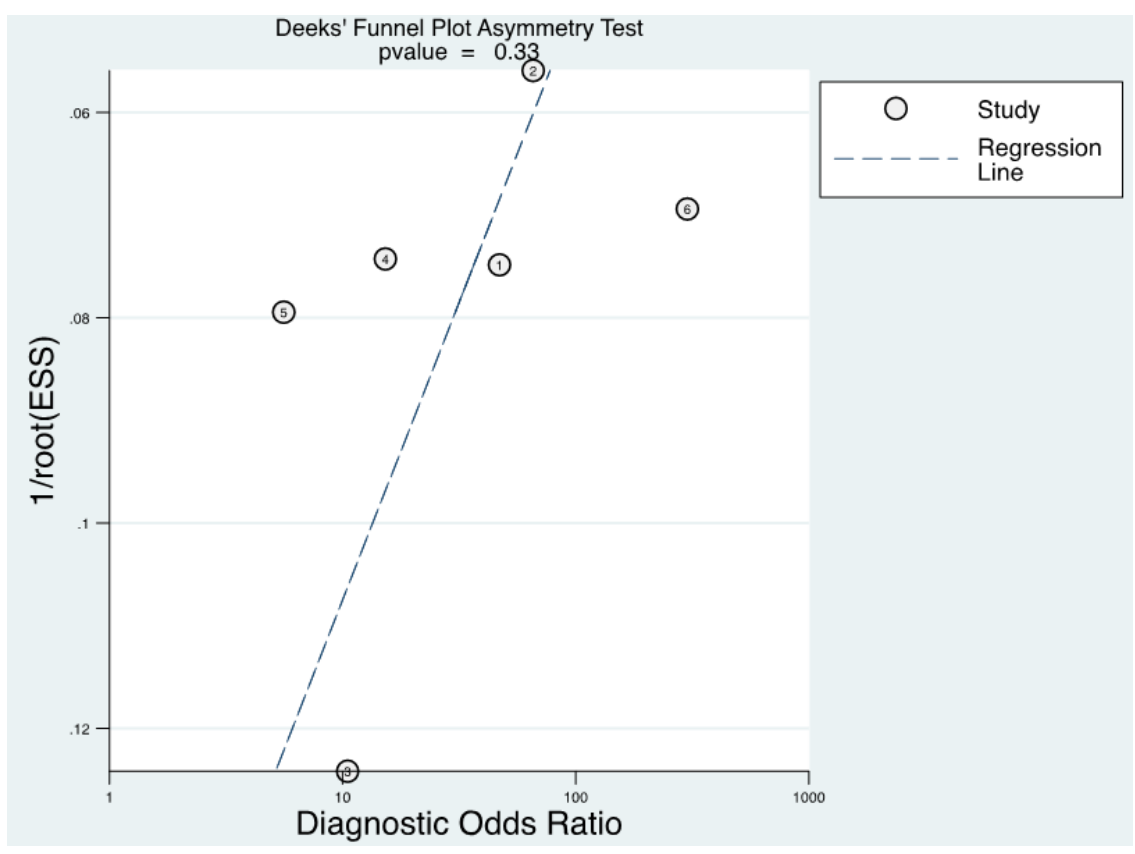

Figure 10: Deeks' funnel plot asymmetry test for publication. 
specificity was explored by univariate meta-regression analysis and subgroup analysis. Urine UCA1 has higher diagnostic accuracy for bladder cancer detection in nonChinese persons than in Chinese persons, indicating that ethnicity may be one of the causes of heterogeneity. Due to there were insufficient eligible studies to fully elucidate the source of the heterogeneity, the possible reason for the heterogeneity need to be investigated in future studies.

The current study has several limitations. First, despite extensive literature search were performed, the number of included studies and sample sizes were small, which may restrict our ability to evaluate the accuracy of urine UCA1. Second, we could not determine the ideal cut-off value for urine UCA1 test, due to different cutoff values were adopted in each study. Third, this metaanalysis was a retrospective analysis, which may limit the conclusion due to selection bias. Fourth, only articles published in English or Chinese were enrolled in our metaanalysis, which may cause inevitable bias.

Despite these limitations, the present evidence suggests that urine UCA1 is potential to be a diagnosis biomarker for bladder cancer, due to this non-invasive method has good overall diagnostic performance. However, large-scale and comprehensive studies must be performed in the future to validate this finding.

\section{MATERIALS AND METHODS}

\section{Search strategy}

This meta-analysis as conducted under the diagnostic meta-analysis guidelines [32]. Studies regarding the diagnostic value of UCA1 in detecting bladder cancer were searched in PubMed, Cochrane library, Chinese Wan Fang and the China National Knowledge Infrastructure (CNKI) databases up to December 2016. Both MeSH terms and free-text words were used in the search strategy to increase sensitivity. The following search keywords were used in combination: "UCA1" or "urothelial carcinoma-associated 1", "bladder cancer" or "bladder carcinoma" or "carcinoma of urinary bladder". In addition, references of all articles in these eligible studies were also read to identify additional relevant literature.

\section{Inclusion and exclusion criteria}

The inclusion criteria were listed as follows: (1) articles were association between UCA1 and bladder cancer; (2) a diagnostic standard of bladder cancer was included; (3) sufficient data (true positive, false positive, false negative and true negative) for calculating sensitivity and specificity; (4) studies should base on humans; (5) studies were published in English or Chinese. Exclusion criteria were as follows: (1) studies without usable or overlapping data; (2) reviews, letters, case report and conference abstracts; (3) insufficient data for calculating sensitivity and specificity.

\section{Data extraction}

Two investigators (Xiangrong Cui and Xuan Jing) retrieved the eligible publications independently. Any disagreement between the two investigators was determined through a discussion with a third investigator. The following data were extracted: first author, year of publication, country, ethnicity of patients, sample size, type of specimen, true and false positive and negative.

\section{Statistical analysis}

For the diagnostic meta-analysis, the accuracy indicator include the pooled sensitivity (SEN), pooled specificity (SPE), positive likelihood ratio (PLR), negative likelihood ration (NLR), diagnostic odds ratio (DOR), and their 95\% confidence interval (CI) were calculated using the random-effect model. PLR was on behalf of the odds of positive test results of bladder cancer patients, while NLR reflected the odds of positive results in those without bladder cancer. DOR was the outcome of the combination of PLR and NLR (DOR = PLR/NLR). Simultaneously, the summary receiver operator characteristic (SROC) curve was created and the under the SROC curve (AUC) was calculated [33]. The analysis of diagnostic accuracy was pursuant to a SROC curve and the AUC of the SROC. In addition, the $I^{2}$ and $Q$ test were performed to evaluate heterogeneity in SEN and SPE among included studies. If the tests show a $P<0.1$ or $I^{2}>50 \%$, the existence of significant heterogeneity would be verified, and then the random-effect model was employed. If not, the fixedeffect model was more appropriate [34, 35]. Subsequently, meta-regression and subgroup analyses were conducted to explore potential sources of between-study heterogeneity. Furthermore, Deeks' funnel plots were adopted to test the publication bias [36]. All statistical analyses were performed using RevMan 5.3 (Revman, the Cochrane Collaboration) and Stata 12.0 (Stata, College Station).

\section{ACKNOWLEDGMENTS}

We are indebted to the donors whose names were not included in the author list, but who participated in this program. This work was supported by the National Natural Science Foundation of China (81670270) and Scientific Research Project of Shanxi Provincial Department of Health (201601070).

\section{CONFLICTS OF INTEREST}

The authors declare no conflicts of interest. 


\section{REFERENCES}

1. Amin MB, Smith SC, Reuter VE, Epstein JI, Grignon DJ, Hansel DE, Lin O, McKenney JK, Montironi R, Paner GP, Al-Ahmadie HA, Algaba F, Ali S, et al. Update for the practicing pathologist: The International Consultation On Urologic Disease-European association of urology consultation on bladder cancer. Modern pathology. 2015; 28:612-630

2. Kang M, Jeong CW, Kwak C, Kim HH, Ku JH. Preoperative neutrophil-lymphocyte ratio can significantly predict mortality outcomes in patients with non-muscle invasive bladder cancer undergoing transurethral resection of bladder tumor. Oncotarget. 2016; 8:12891-12901. doi: 10.18632/oncotarget.14179.

3. Sekar V, Mehrotra DG, Majumder B. Molecular and Functional Diagnostic Tools in Precision Oncology for Urological Malignancies. Indian journal of surgical oncology. 2017; 8:24-32.

4. Cui L, Sun MM, Zhao ZH, Yang JP, Zheng YP, He LL, Chen KS, Fan QX. BLCA-4, UBC combined detection for early diagnosis of bladder cancer. Journal of biological regulators and homeostatic agents. 2016; 30:485-490.

5. Urquidi V, Netherton M, Gomes-Giacoia E, Serie DJ, Eckel-Passow J, Rosser CJ, Goodison S. A microRNA biomarker panel for the non-invasive detection of bladder cancer. Oncotarget. 2016; 7:86290-86299. doi: 10.18632/ oncotarget.13382.

6. Wiener HG, Mian C, Haitel A, Pycha A, Schatzl G, Marberger M. Can urine bound diagnostic tests replace cystoscopy in the management of bladder cancer? The Journal of urology. 1998; 159:1876-1880.

7. Rink M, Babjuk M, Catto JW, Jichlinski P, Shariat SF, Stenzl A, Stepp H, Zaak D, Witjes JA. Hexyl aminolevulinate-guided fluorescence cystoscopy in the diagnosis and follow-up of patients with non-muscleinvasive bladder cancer: a critical review of the current literature. European urology. 2013; 64:624-638.

8. Eissa S, Kassim S, El-Ahmady O. Detection of bladder tumours: role of cytology, morphology-based assays, biochemical and molecular markers. Current opinion in obstetrics \& gynecology. 2003; 15:395-403.

9. Shao Y, Zhang Y, Hou Y, Tong H, Zhuang R, Ji Z, Wang B, Zhou Y, Lu W. A novel long noncoding RNA PILRLS promote proliferation through TCL1A by activing MDM2 in Retroperitoneal liposarcoma. Oncotarget. 2017; 8:1397113978. doi: 10.18632/oncotarget.14814.

10. Lanzos A, Carlevaro-Fita J, Mularoni L, Reverter F, Palumbo E, Guigo R, Johnson R. Discovery of Cancer Driver Long Noncoding RNAs across 1112 Tumour Genomes: New Candidates and Distinguishing Features. Scientific reports. 2017; 7:41544.

11. Bao Z, Zhang W, Dong D. A potential prognostic lncRNA signature for predicting survival in patients with bladder urothelial carcinoma. Oncotarget. 2017; 8:10485-10497. doi: 10.18632/oncotarget.14441.

12. Chen R, Wang G, Zheng Y, Hua Y, Cai Z. Long non-coding RNAs in osteosarcoma. Oncotarget. 2017; 8:20462-20475. doi: 10.18632/oncotarget.14726.

13. Cheng Z, Bai Y, Wang P, Wu Z, Zhou L, Zhong M, Jin Q, Zhao J, Mao H, Mao H. Identification of long noncoding RNAs for the detection of early stage lung squamous cell carcinoma by microarray analysis. Oncotarget. 2017; 8:13329-13337. doi: 10.18632/oncotarget.14522.

14. XianGuo C, ZongYao H, Jun Z, Song F, GuangYue L, LiGang Z, KaiPing Z, YangYang Z, ChaoZhao L. Promoting progression and clinicopathological significance of NEAT1 over-expression in bladder cancer. Oncotarget. 2016 Jun 15. doi: 10.18632/oncotarget.10084. [Epub ahead of print].

15. Duan W, Du L, Jiang X, Wang R, Yan S, Xie Y, Yan K, Wang Q, Wang L, Zhang X, Pan H, Yang Y, Wang C. Identification of a serum circulating lncRNA panel for the diagnosis and recurrence prediction of bladder cancer. Oncotarget. 2016; 7:78850-78858. doi: 10.18632/ oncotarget. 12880 .

16. Zhou M, Diao Z, Yue X, Chen Y, Zhao H, Cheng L, Sun J. Construction and analysis of dysregulated lncRNAassociated ceRNA network identified novel lncRNA biomarkers for early diagnosis of human pancreatic cancer. Oncotarget. 2016; 7:56383-56394. doi: 10.18632/ oncotarget.10891.

17. Zhu H, Zhang L, Yan S, Li W, Cui J, Zhu M, Xia N, Yang Y, Yuan J, Chen X, Luo J, Chen R, Xing R, et al. LncRNA16 is a potential biomarker for diagnosis of early-stage lung cancer that promotes cell proliferation by regulating the cell cycle. Oncotarget. 2017; 8:7867-7877. doi: 10.18632/oncotarget.13980.

18. Wang XS, Zhang Z, Wang HC, Cai JL, Xu QW, Li MQ, Chen YC, Qian XP, Lu TJ, Yu LZ, Zhang Y, Xin DQ, Na YQ, et al. Rapid identification of UCA1 as a very sensitive and specific unique marker for human bladder carcinoma. Clinical cancer research. 2006; 12:4851-4858.

19. Milowich D, Le Mercier M, De Neve N, Sandras F, Roumeguere T, Decaestecker C, Salmon I, Rorive S. Diagnostic value of the UCA1 test for bladder cancer detection: a clinical study. SpringerPlus. 2015; 4:349.

20. Eissa S, Matboli M, Essawy NO, Shehta M, Kotb YM. Rapid detection of urinary long non-coding RNA urothelial carcinoma associated one using a PCR-free nanoparticlebased assay. Biomarkers. 2015; 20:212-217.

21. Srivastava AK, Singh PK, Rath SK, Dalela D, Goel MM, Bhatt ML. Appraisal of diagnostic ability of UCA1 as a biomarker of carcinoma of the urinary bladder. Tumour biology. 2014; 35:11435-11442.

22. Eissa S, Matboli M, Essawy NO, Kotb YM. Integrative functional genetic-epigenetic approach for selecting genes as urine biomarkers for bladder cancer diagnosis. Tumour 
biology. 2015; 36:9545-9552.

23. Zhang Z, Hao H, Zhang CJ, Yang XY, He Q, Lin J. [Evaluation of novel gene UCA1 as a tumor biomarker for the detection of bladder cancer]. [Article in Chinese]. Zhonghua yi xue za zhi. 2012; 92:384-387.

24. Li F, Li X, Wang F, Zhao L, Chen W. Establishment of method about detecting UCA1 mRNA through realtime fluorescence quantitative PCR reverse transcription polymerase chain reaction and its clinical assessment. Journal of Molecular Diagnosis and Therapy. 2012; 04:171176.

25. Mukherjee N, Cardenas E, Bedroll R, Ghosh R. SETD6 regulates NF-kappaB signaling in urothelial cell survival: Implications for bladder cancer. Oncotarget. 2017; 8:1511415125. doi: 10.18632/oncotarget.14750.

26. Lu J, Zheng X, Li F, Yu Y, Chen Z, Liu Z, Wang Z, Xu $\mathrm{H}$, Yang W. Tunneling nanotubes promote intercellular mitochondria transfer followed by increased invasiveness in bladder cancer cells. Oncotarget. 2017; 8:15539-15552. doi: 10.18632/oncotarget.14695.

27. Segovia C, Martinez-Fernandez M, Duenas M, Rubio C, Lopez-Calderon FF, Costa C, Saiz-Ladera C, FernandezGrajera M, Duarte J, Garcia Munoz H, de la Rosa F, Villacampa F, Castellano D, et al. Opposing roles of PIK3CA gene alterations to EZH2 signaling in non-muscle invasive bladder cancer. Oncotarget. 2017; 8:10531-10542. doi: 10.18632/oncotarget.14453.

28. Kamat AM, Hahn NM, Efstathiou JA, Lerner SP, Malmstrom PU, Choi W, Guo CC, Lotan Y, Kassouf W. Bladder cancer. Lancet. 2016; 388:2796-2810.

29. Olivieri M, Ferro M, Terreri S, Durso M, Romanelli A, Avitabile C, De Cobelli O, Messere A, Bruzzese D, Vannini I, Marinelli L, Novellino E, Zhang W, et al. Long non- coding RNA containing ultraconserved genomic region 8 promotes bladder cancer tumorigenesis. Oncotarget. 2016; 7:20636-20654. doi: 10.18632/oncotarget.7833.

30. Kumar P, Nandi S, Tan TZ, Ler SG, Chia KS, Lim WY, Butow Z, Vordos D, De la Taille A, Al-Haddawi M, Raida M, Beyer B, Ricci E, et al. Highly sensitive and specific novel biomarkers for the diagnosis of transitional bladder carcinoma. Oncotarget. 2015; 6:13539-13549. doi: 10.18632/oncotarget.3841.

31. Coory MD. Comment on: Heterogeneity in metaanalysis should be expected and appropriately quantified. International journal of epidemiology. 2010; 39:932; author reply 933.

32. Leeflang MM, Deeks JJ, Gatsonis C, Bossuyt PM, Cochrane Diagnostic Test Accuracy Working G. Systematic reviews of diagnostic test accuracy. Annals of internal medicine. 2008; 149:889-897.

33. Moses LE, Shapiro D, Littenberg B. Combining independent studies of a diagnostic test into a summary ROC curve: data-analytic approaches and some additional considerations. Statistics in medicine. 1993; 12:1293-1316.

34. Higgins JP, Thompson SG, Deeks JJ, Altman DG. Measuring inconsistency in meta-analyses. Bmj. 2003; 327:557-560.

35. Dinnes J, Deeks J, Kirby J, Roderick P. A methodological review of how heterogeneity has been examined in systematic reviews of diagnostic test accuracy. Health technology assessment. 2005; 9:1-113, iii.

36. Deeks JJ, Macaskill P, Irwig L. The performance of tests of publication bias and other sample size effects in systematic reviews of diagnostic test accuracy was assessed. Journal of clinical epidemiology. 2005; 58:882-893. 